\title{
Modeling and numerical simulation of particulate flows by the Eulerian-Lagrangian approach
}

\author{
N. A. Patankar* and D. D. Joseph \\ Department of Aerospace Engineering and Mechanics, University of Minnesota, Minneapolis, MN 55455
}

\begin{abstract}
In this paper we present an Eulerian-Lagrangian numerical simulation (LNS) scheme for particulate flows. The overall algorithm in the present approach is a variation of the scheme presented earlier by N. Patankar and Joseph (1999). In this numerical scheme we solve the fluid phase continuity and momentum equations on an Eulerian grid. The particle motion is governed by Newton's law thus following the Lagrangian approach. Momentum exchange from the particle to fluid is modeled in the fluid phase momentum equation. Forces acting on the particles include drag from the fluid, body force and the interparticle force that prevents the particle volume fraction from exceeding the closepacking limit. There is freedom to use different models for these forces and to introduce other forces. In this paper we have used two types of interparticle forces. The effect of viscous stresses are included in the fluid phase equations. The volume fraction of the particles appear in the fluid phase continuity and momentum equations. The fluid and particle momentum equations are coupled in the solution procedure unlike the earlier approach of N. Patankar and Joseph (1999). A finite volume method is used to solve these equations on an Eulerian grid. Particle positions are updated explicitly. This numerical scheme can handle a range of particle loadings and particle types.

We solve the fluid phase continuity and momentum equations using a Chorin-type fractional-step method. The numerical scheme is verified by comparing results with test cases and experiments.
\end{abstract}

Key Words: Two-phase flow, Eulerian-Lagrangian numerical simulation (LNS), multiphase particle-in-cell (MP-PIC) method, particulate flows, Chorin scheme,

\footnotetext{
${ }^{*}$ Current address: Department of Mechanical Engineering, Northwestern University, Evanston, IL 60208.
} 
fractional-step method, non-staggered grid, bimodal sedimentation, inclined sedimentation, gas-solid and liquid-solid fluidization.

\section{Introduction}

Numerical schemes based on mathematical models of separated particulate multiphase flow have used the continuum approach for all the phases or a continuum approach for the fluid phase and a Lagrangian approach for the particles. These simulation methods can be applied in various settings; e.g. sedimenting and fluidized suspensions, lubricated transport, hydraulic fracturing of reservoirs, slurries, sprays etc.

Continuum-continuum (or Eulerian-Eulerian) approach considers the particulate phase to be a continuous fluid interpenetrating and interacting with the fluid phase (Gidaspow 1994). In the Eulerian-Lagrangian formulation each computational particle (called parcel) is considered to represent a group of particles interacting with the fluid and possessing the same characteristics such as size, composition etc. Positions of these parcels are then calculated using Newton's equations of motion. It has been found that the required number of parcels to accurately represent the particle phase is not excessive (Dukowicz 1980).

The Eulerian-Lagragian numerical simulation methods have been developed with different assumptions. In problems such as the dispersion of atmospheric pollutants, it may be assumed that the particles do not perturb the flow field. The solution then involves tracing the particle trajectories in a known velocity field i.e. the fluid phase equations are solved independent of the particles (Gauvin, Katta and Knelman 1975). In other problems the particles may carry sufficient momentum to set the surrounding fluid in motion. In this case it is necessary to include the fluid-particle momentum exchange term in the fluid phase equation. However, the volume occupied by the particles in a computational cell in comparison with the volume of the fluid may still be neglected (Crowe, Sharma and Stock 1977). When the particle volume is significant it is important to model the volume fraction in both the momentum and continuity equations (Dukowicz 1980). At high particle volume fractions the effective viscosity of the suspension is high 
and this effect should be ideally modeled through the viscous stress term in the governing equations.

Andrews and O'Rourke (1996) and Snider, O'Rourke and Andrews (1998) presented a multiphase particle-in-cell (MP-PIC) method for particulate flows that accounts for full coupling between the fluid and particle phase as well as the interparticle stress to prevent the particle volume from exceeding the close packing limit. The fluid phase is assumed to be inviscid where viscosity is significant on the scale of the particles and is used only in the particle drag formula. In this approach the particle phase is considered both as a continuum and as a discrete phase. Interparticle stresses are calculated by treating the particles as a continuum phase. Particle properties are mapped to and from an Eulerian grid. Continuum derivatives that treat the particle phase as a fluid are evaluated to model interparticle stress and then mapped back to the individual particles. This results in a computational method for multiphase flows that can handle particulate loading ranging from dense to dilute and for particles of different sizes and materials. N. Patankar and Joseph (1999) presented a new Eulerian-Lagrangian numerical simulation (LNS) scheme for particulate flows in three-dimensional geometries. They apply the MP-PIC approach of Andrews and O'Rourke (1996).

The hydrodynamic force acting on the particle surface represents the fluid-particle momentum exchange. Different models can be used for the hydrodynamic force on the particle in the Eulerian-Lagrangian approach. These models can be developed through experimental investigation. At the same time, development of direct numerical simulation (DNS) techniques for rigid particulate flows (e.g. Hu, Crochet and Joseph 1992, Hu 1996, Johnson and Tezduyar 1997, Glowinski, Pan, Hesla and Joseph 1999, N. Patankar, Singh, Joseph, Glowinski and Pan 2000) have provided an invaluable tool for modeling the hydrodynamic force in many applications. It would be straightforward to use these models for the hydrodynamic force from experiments or DNS results, when available, in the LNS technique.

The primary objective of this work is to develop a model-based numerical simulation scheme for particulate flows that has the following features: (a) A flexibility to use different models for the fluid-particle force (drag and lift) and the effective properties (such as the effective viscosity) of the suspension. Such models are expected to be 
developed from our effort on the direct numerical simulation (DNS) of particulate flows. (b) A capability to simulate the motion of particles of different sizes and material properties. (c) A capability to handle dense particulate flows without exceeding the closepacking limit. (d) An efficient numerical algorithm capable of quick computations at each time-step with modest memory requirements as compared to the direct numerical simulation methods. The other objective is to suggest a model for the viscous stress terms in the fluid phase equations. We choose an Eulerian-Lagrangian numerical simulation method because the models developed from DNS can be most naturally introduced in this approach. Additional continuity and momentum equations for each particulate phase are not required for multimodal simulations. The Lagrangian tracking of particles naturally predict the unstable flow features without complexities in the numerical implementation.

The numerical algorithm used in this paper is a variation of the scheme by $\mathrm{N}$. Patankar and Joseph (1999). We solve the fluid phase continuity and momentum equations using a Chorin-type (Chorin 1968) fractional-step method, but unlike the approach of N. Patankar and Joseph (1999) we solve the particle momentum equation coupled with the fluid phase equations. As a result the pressure equation set up in this approach is different from the one used by them. A non-staggered grid for velocity and pressure (Rhie and Chow 1982) is used.

In the MP-PIC and LNS formulation of N. Patankar and Joseph (1999) a particle stress term that acts like pressure is used to prevent the particle volume fraction from exceeding the close-packing limit. This stress model introduces a repelling force on particles moving into regions where the particle volume fraction is large. In this paper we use this model for some problems. We also use a different "collision" scheme that prevents the particle volume fraction from exceeding the close packing limit. We introduce a parcel-parcel and parcel-wall collision force to ensure that there is no overlap between any two parcel regions and between the parcel regions and the wall. Consequently the particle volume fraction does not exceed the close-packing limit in any part of the computational domain. We have used this scheme for the simulation of bubbling fluidization of gas-particle and liquid-particle mixtures.

In the next section we will present governing equations for the Eulerian-Lagrangian formulation. In section 3 the numerical scheme will be explained. This computational 
scheme will then be verified in section 4 by comparing results with test cases and experiments. Simulation results for bimodal sedimentation, inclined sedimentation and bubbling fluidization of gas-particle and liquid-particle mixtures are presented. Conclusions will be stated in section 5 .

\section{Governing equations}

We use averaged equations of motion for the fluid phase (see Joseph and Lundgren 1990 and references therein). Continuity equation is given by

$$
\frac{\partial}{\partial \mathrm{t}}\left(\rho_{\mathrm{f}} \theta_{\mathrm{f}}\right)+\nabla \cdot\left(\rho_{\mathrm{f}} \theta_{\mathrm{f}} \mathbf{u}_{\mathrm{f}}\right)=0
$$

where $\rho_{\mathrm{f}}$ is the density of the suspending fluid, $\theta_{\mathrm{f}}$ is the fluid volume fraction and $\mathbf{u}_{\mathrm{f}}$ is the average velocity of the fluid phase. The average velocity field of the fluid phase does not satisfy the divergence-free condition even if we consider an incompressible suspending fluid. The momentum equation of the fluid phase can be obtained based on ensemble averaging. Using the "small particle approximation" Zhang and Prosperetti (1997) obtained the following form for the ensemble averaged momentum equation of the fluid phase

$$
\begin{aligned}
& \frac{\partial}{\partial \mathrm{t}}\left(\rho_{\mathrm{f}} \theta_{\mathrm{f}} \mathbf{u}_{\mathrm{f}}\right)+\nabla \cdot\left(\rho_{\mathrm{f}} \theta_{\mathrm{f}} \mathbf{u}_{\mathrm{f}} \mathbf{u}_{\mathrm{f}}\right)=-\nabla\left(\theta_{\mathrm{f}} \mathrm{p}\right)+\nabla \cdot\left[\mu_{\mathrm{f}} \mathbf{D}_{\mathrm{m}}\right] \\
& +\nabla \cdot \mathbf{S}_{\mathrm{p}}-\mathbf{F}+\nabla \cdot \mathbf{M}_{\mathrm{r}}+\nabla \cdot \mathbf{S}_{\mathrm{m}},
\end{aligned}
$$

where $p$ is the average dynamic pressure in the fluid phase, $\mu_{\mathrm{f}}$ is the viscosity of the fluid phase, $\quad \mathbf{D}_{\mathrm{m}}=\nabla \mathbf{u}_{\mathrm{m}}+\nabla \mathbf{u}_{\mathrm{m}}{ }^{\mathrm{T}}$ is the average deformation-rate of the fluid-particle composite, $\mathbf{u}_{\mathrm{m}}$ is the composite velocity of the mixture, $\mathbf{S}_{\mathrm{p}}$ is the "stresslet-torque" per unit volume acting on the fluid phase, $\mathbf{F}$ is the rate of momentum exchange per unit volume between the fluid and particle phases, $\mathbf{M}_{\mathrm{r}}$ is the Reynolds stress contribution and $\mathbf{S}_{\mathrm{m}}$ is the contribution to stress due to higher moments of the surface traction about the 
particle center. Eqn. (2) is applicable to suspensions of rigid particles in incompressible Newtonian fluids. Gravity is balanced by the hydrostatic pressure and does not arise explicitly in the momentum equation. In the present work we neglect the last two terms on the right hand side of (2). Modeling of these terms is relegated to our future effort. Following the analysis of Zhang and Prosperetti (1997) it can be verified that in the dilute and zero Reynolds number limit $-\theta_{\mathrm{f}} \mathrm{p} \mathbf{I}+\mu_{\mathrm{f}} \mathbf{D}_{\mathrm{m}}+\mathbf{S}_{\mathrm{p}}$ becomes $-\mathrm{p} \mathbf{I}+\mu^{*} \mathbf{D}_{\mathrm{m}}$, where $\mathbf{I}$ is the identity tensor and $\mu^{*}$ is the well-known effective viscosity of a dilute suspension of rigid particles by Einstein (Batchelor 1967). We generalize this term to non-dilute suspensions by using the Thomas (1965) correlation for the effective viscosity (valid at low Reynolds numbers)

$$
\mu^{*}=\mu_{\mathrm{f}}\left(1+2.5 \theta_{\mathrm{f}}+10.05 \theta_{\mathrm{f}}^{2}+0.00273 \mathrm{e}^{16.6 \theta_{\mathrm{f}}}\right)
$$

The effect of the Reynolds number and the particle shape and configuration should enter into a general expression for the flow resistance. We use (3) since appropriate models that account for these effects are not yet available. For typical cases considered in our simulations, the high volume fraction region (in which the effective viscosity is high) is observed near the walls. The velocity in these regions is small (resulting in a low Reynolds number locally) due to the no-slip condition at the walls and the viscous effects. Joseph and Lundgren (1990) compared the mixture theory equations (e.g. Nunziato, Passman, Givler, MacTigue and Brady 1986) for the fluid phase with those obtained from ensemble averaging. They noted that the fluid phase equations based on mixture theory had the term $\theta_{\mathrm{f}}\left(\nabla \mathbf{u}_{\mathrm{f}}+\nabla \mathbf{u}_{\mathrm{f}}{ }^{\mathrm{T}}\right)$ in place of $\mathbf{D}_{\mathrm{m}}$. These two expressions differ by terms of the order of particle volume fraction (Zhang and Prosperetti 1997). We choose to use the former expression in place of $\mathbf{D}_{\mathrm{m}}$ since it is more convenient to implement in the numerical solution procedure. The fluid phase momentum equation is then given by 


$$
\begin{aligned}
& \frac{\partial}{\partial \mathrm{t}}\left(\rho_{\mathrm{f}} \theta_{\mathrm{f}} \mathbf{u}_{\mathrm{f}}\right)+\nabla \cdot\left(\rho_{\mathrm{f}} \theta_{\mathrm{f}} \mathbf{u}_{\mathrm{f}} \mathbf{u}_{\mathrm{f}}\right)=-\nabla \mathrm{p}+\nabla \cdot\left[\theta_{\mathrm{f}} \mu^{*} \nabla \mathbf{u}_{\mathrm{f}}\right] \\
& +\nabla \cdot\left[\theta_{\mathrm{f}} \mu^{*} \nabla \mathbf{u}_{\mathrm{f}}^{\mathrm{T}}\right]-\mathbf{F}
\end{aligned}
$$

where $\mu^{*}$ is given by (3). Expression for $\mathbf{F}$ will be presented shortly.

The evolution of the particle phase is governed by a Liouville equation for the particle distribution function $\phi\left(\mathbf{x}_{\mathrm{p}}, \mathbf{u}_{\mathrm{p}}, \rho_{\mathrm{p}}, \mathrm{V}_{\mathrm{p}}, \mathrm{t}\right)$

$$
\frac{\partial \phi}{\partial \mathrm{t}}+\nabla_{\mathbf{x}} \cdot\left(\phi \mathbf{u}_{\mathrm{p}}\right)+\nabla_{\mathbf{u}_{\mathrm{p}}} \cdot\left(\phi \mathbf{A}_{\mathrm{p}}\right)=0
$$

where $\mathbf{x}_{\mathrm{p}}$ is the particle position, $\mathbf{u}_{\mathrm{p}}$ is the particle velocity, $\rho_{\mathrm{p}}$ is the particle density and $\mathrm{V}_{\mathrm{p}}$ is the particle volume. $\mathbf{A}_{\mathrm{p}}$ is the particle acceleration given by

$$
\begin{aligned}
\mathbf{A}_{\mathrm{p}}=\frac{\mathrm{d} \mathbf{u}_{\mathrm{p}}}{\mathrm{dt}} & =\mathrm{D}_{\mathrm{p}}\left(\mathbf{u}_{\mathrm{f}}-\mathbf{u}_{\mathrm{p}}\right)-\frac{1}{\rho_{\mathrm{p}}} \nabla \mathrm{p}_{\mathrm{p}}+\frac{1}{\rho_{\mathrm{p}}} \nabla \cdot\left(\mu_{\mathrm{f}}\left[\nabla \mathbf{u}_{\mathrm{f}}+\nabla \mathbf{u}_{\mathrm{f}}{ }^{\mathrm{T}}\right]\right)_{\mathrm{p}} \\
& +\left(1-\frac{\rho_{\mathrm{f}}}{\rho_{\mathrm{s}}}\right) \mathbf{g}+\mathbf{A}_{\mathrm{cp}},
\end{aligned}
$$

where $\theta_{\mathrm{s}}$ is the particle volume fraction, $\nabla \mathrm{p}_{\mathrm{p}}$ denotes fluid pressure gradient at the particle location and $\nabla \cdot\left(\mu_{\mathrm{f}}\left[\nabla \mathbf{u}_{\mathrm{f}}+\nabla \mathbf{u}_{\mathrm{f}}{ }^{\mathrm{T}}\right]\right)_{\mathrm{p}}$ denotes a force on the particles due the gradient of viscous stress in the fluid phase. Equation (6) models acceleration due to hydrodynamic drag, dynamic pressure gradient, gradient of viscous stress in the fluid phase, net buoyant force and interparticle collision $\left(\mathbf{A}_{\mathrm{cp}}\right)$. Using (6) in (5) one can derive the average momentum equation of the particle phase. It can be easily verified that the particle phase equations are in the same form as that given by Zhang and Prosperetti (1997). Different models for force on the particle can also be used in (6). In the present work we neglect the force on the particles due to gradient of viscous stress in the fluid phase. Modeling of acceleration due interparticle collision enables simulation of dense particulate flows; details of which will be given in the next section. Following the 
analysis of Gibilaro, Di Felice, Waldram and Foscolo (1985) we use the following expression for $D_{p}$

$$
\mathrm{D}_{\mathrm{p}}=\mathrm{C}_{\mathrm{d}} \frac{3}{8} \frac{\rho_{\mathrm{f}}}{\rho_{\mathrm{p}}} \frac{\left|\mathbf{u}_{\mathrm{f}}-\mathbf{u}_{\mathrm{p}}\right|}{\mathrm{R}_{\mathrm{p}}},
$$

where $C_{d}$ is given by (Rowe 1961)

$$
\begin{aligned}
\mathrm{C}_{\mathrm{d}} & =\frac{24}{\operatorname{Re}}\left(1+0.15 \mathrm{Re}^{0.687}\right) \theta_{\mathrm{f}}^{-1.8} & \text { for } & \operatorname{Re}<1000, \\
& =0.44 \theta_{\mathrm{f}}^{-1.8} & \text { for } & \operatorname{Re} \geq 1000,
\end{aligned}
$$

and

$$
\mathrm{Re}=\frac{2 \rho_{\mathrm{f}} \theta_{\mathrm{f}}\left|\mathbf{u}_{\mathrm{f}}-\mathbf{u}_{\mathrm{p}}\right| \mathrm{R}_{\mathrm{p}}}{\mu_{\mathrm{f}}}, \mathrm{R}_{\mathrm{p}}=\left(\frac{3 \mathrm{~V}_{\mathrm{p}}}{4 \pi}\right)^{1 / 3} .
$$

$C_{d}$ is the drag coefficient, $R e$ is the Reynolds number and $R_{p}$ is the particle radius (we assume spherical particles). In (7) we have approximated the velocity of the particle with respect to the composite by $\theta_{\mathrm{f}}\left(\mathbf{u}_{\mathrm{f}}-\mathbf{u}_{\mathrm{p}}\right)$. The expression for drag in (7) is similar to that by Andrews and O'Rourke (1996). The particle volume fraction $\theta_{\mathrm{s}}$ is defined by

$$
\theta_{\mathrm{s}}=\iiint \phi \mathrm{V}_{\mathrm{p}} \mathrm{dV} \mathrm{V}_{\mathrm{p}} \mathrm{d} \rho_{\mathrm{p}} \mathrm{d} \mathbf{u}_{\mathrm{p}} .
$$

Fluid volume fraction $\theta_{\mathrm{f}}$ is then given by

$$
\theta_{\mathrm{f}}=1-\theta_{\mathrm{s}} .
$$

The interphase momentum transfer function $\mathbf{F}$ is given by

$$
\mathbf{F}=\iiint \phi \mathrm{V}_{\mathrm{p}} \rho_{\mathrm{p}}\left[\mathrm{D}_{\mathrm{p}}\left(\mathbf{u}_{\mathrm{f}}-\mathbf{u}_{\mathrm{p}}\right)-\frac{1}{\rho_{\mathrm{p}}} \nabla \mathrm{p}_{\mathrm{p}}+\frac{1}{\rho_{\mathrm{p}}} \nabla \cdot\left(\mu_{\mathrm{f}}\left[\nabla \mathbf{u}_{\mathrm{f}}+\nabla \mathbf{u}_{\mathrm{f}}{ }^{\mathrm{T}}\right]\right)_{\mathrm{p}}\right] \mathrm{dV} \mathrm{p} \mathrm{d} \rho_{\mathrm{p}} \mathrm{d} \mathbf{u}_{\mathrm{p}} .
$$

Note that in the present work we neglect the third term in the integral in (10). Andrews and O'Rourke (1996) showed that the Lagrangian formulation above accounts for the 
kinematic stress that arises from local particle velocity fluctuations about the mean velocity.

\section{Numerical scheme}

We use a finite-volume method on a three-dimensional Eulerian grid to solve the fluid phase equations in cartesian coordinate system. A non-staggered grid for velocity and pressure (Rhie and Chow 1982) is used. The particle phase equations are solved by considering the motion of a finite number of computational particles which represent a sample of the total population of particles. Each computational particle, henceforth referred to as a parcel, is considered to represent a group of particles of identical size, velocity and position.

\subsection{Interpolation scheme}

In order solve the particle equation of motion it is necessary to interpolate variables to the particle position. Similarly the solution of fluid phase equations requires the calculation of variables on the Eulerian grid. This requires the interpolation of these variables from particle location to the Eulerian grid. This is accomplished by using bilinear interpolation function formed from the product of linear interpolation functions in the $\mathrm{x}, \mathrm{y}$ and $\mathrm{z}$ directions (Snider et al. 1998). The bilinear interpolation function $\mathrm{S}_{\mathrm{ijk}}(\mathbf{x})$ is unity at a given grid node $(\mathrm{i}, \mathrm{j}, \mathrm{k})$ which is at the cell center and decreases to zero at the 26 neighboring nodes and the domain beyond these neighboring nodes. The position $\mathbf{x}_{\mathrm{p}}$ of any particle can be located in a box defined by eight Eulerian grid nodes surrounding it. The sum of the eight interpolation functions, due to the surrounding nodes, at a particle location is unity.

The particle volume fraction on the Eulerian grid is calculated by

$$
\theta_{\text {sijk }}=\frac{1}{\mathrm{~V}_{\mathrm{ijk}}} \sum_{\mathrm{p}} \mathrm{N}_{\mathrm{p}} \mathrm{V}_{\mathrm{p}} \mathrm{S}_{\mathrm{ijk}}\left(\mathbf{x}_{\mathrm{p}}\right),
$$


where $\theta_{\mathrm{sijk}}$ is the particle volume fraction at grid node $(\mathrm{i}, \mathrm{j}, \mathrm{k}), \mathrm{V}_{\mathrm{ijk}}$ is the volume of the Eulerian cell $(\mathrm{i}, \mathrm{j}, \mathrm{k})$ and $\mathrm{N}_{\mathrm{p}}$ is the number of particles in a parcel. Fluid volume $\theta_{\text {fijk }}$ at grid node $(\mathrm{i}, \mathrm{j}, \mathrm{k})$ immediately follows from $(9)$.

Interpolation of a variable from the grid node to particle position is represented by the following example for fluid velocity $\mathbf{u}_{\mathrm{fp}}$ at the particle location:

$$
\mathbf{u}_{\mathrm{fp}}=\sum_{\zeta=1}^{8} \mathrm{~S}_{\varsigma}\left(\mathbf{x}_{\mathrm{p}}\right) \mathbf{u}_{\mathrm{f} \zeta},
$$

where $\zeta$ is an index for the eight grid nodes bounding the particle.

The interphase momentum transfer $\mathbf{F}_{\mathrm{ijk}}$ at a grid node $(\mathrm{i}, \mathrm{j}, \mathrm{k})$ is evaluated by an interpolation scheme given by Snider et al. 1998. According to this scheme the expression for $\mathbf{F}_{\mathrm{ijk}}$ is given by

$$
\begin{aligned}
\mathbf{F}_{\mathrm{ijk}} & =\frac{1}{\mathrm{~V}_{\mathrm{ijk}}} \sum_{\mathrm{p}}\left\{\rho_{\mathrm{p}} \mathrm{V}_{\mathrm{p}} \mathrm{N}_{\mathrm{p}} \mathrm{S}_{\mathrm{ijk}}\left(\mathbf{x}_{\mathrm{p}}\right)\left[\mathrm{D}_{\mathrm{p}}\left(\mathbf{u}_{\mathrm{fp}}-\mathbf{u}_{\mathrm{p}}\right)-\frac{1}{\rho_{\mathrm{p}}} \nabla \mathrm{p}_{\mathrm{p}}\right]\right\} \\
& =\frac{1}{\mathrm{~V}_{\mathrm{ijk}}} \sum_{\mathrm{p}}\left\{\rho_{\mathrm{p}} \mathrm{V}_{\mathrm{p}} \mathrm{N}_{\mathrm{p}} \mathrm{S}_{\mathrm{ijk}}\left(\mathbf{x}_{\mathrm{p}}\right)\left[\mathrm{D}_{\mathrm{p}}\left(\mathbf{u}_{\mathrm{fijk}}-\mathbf{u}_{\mathrm{p}}\right)-\frac{1}{\rho_{\mathrm{p}}} \nabla \mathrm{p}_{\mathrm{ijk}}\right]\right\}
\end{aligned}
$$

This gives a less diffusive interpolation scheme and increases the diagonal dominance of the momentum equation of the fluid phase.

\subsection{Collision scheme}

Numerical simulation scheme for dense particulate flows should ensure that the particle volume fraction does not exceed the close-packing limit (usually taken to be 0.6 in a three-dimensional case) in any part of the computational domain. In the EulerianEulerian approach for two-phase flows this is ensured by a force due to the gradient of interparticle stress in the averaged momentum equation for the particle phase (Gidaspow 1994). Same model was used in the Eulerian-Lagrangian approach by Andrews and 
O'Rourke (1996) and N. Patankar and Joseph (1999). We use this model (henceforth referred to as Model A) to get the following expression for $\mathbf{A}_{\mathrm{cp}}$

$$
\mathbf{A}_{\mathrm{cp}}=-\frac{1}{\theta_{\mathrm{s}} \rho_{\mathrm{p}}} \nabla \tau,
$$

where $\tau$ is the interparticle stress that provides a pressure type force that prevents packing of particles beyond the close-packing limit. $\tau$ is modeled by (Harris and Crighton 1994)

$$
\tau=\frac{\mathrm{P}_{\mathrm{s}} \theta_{\mathrm{s}}^{\beta}}{\theta_{\mathrm{cs}}-\theta_{\mathrm{s}}},
$$

where $P_{s}$ has units of pressure, $\theta_{\mathrm{cs}}$ is the particle volume fraction at close packing and $\beta$ is a constant. A discussion of the factors entering into the choice of $P_{s}$ and $\beta$ is given by Snider et al. (1998). In (15) it is assumed that acceleration of a particle due to interparticle stress is independent of its size and velocity.

In this paper we also use an alternate collision model (henceforth referred to as Model B) that prevents the particle volume fraction from exceeding the close packing limit. Model B is more convenient for applications such as the bubbling fluidized bed. We assume that in a general three-dimensional case the parcel occupies a spherical region in the computational domain. The volume of this spherical region is equal to the total volume of that parcel. The effective radius $\mathrm{R}_{\mathrm{ep}}$ of the spherical region occupied by parcel $\mathrm{p}$ is then given by

$$
\mathrm{R}_{\mathrm{ep}}=\left(\frac{3 \mathrm{~N}_{\mathrm{p}} \mathrm{V}_{\mathrm{p}}}{4 \pi}\right)^{1 / 3} .
$$

We introduce a parcel-parcel and parcel-wall collision force to ensure that there is no overlap between any two parcel regions and between the parcel regions and the wall. As a result, the particle volume fraction will never exceed the close-packing limit in any part of the computational domain. We model the parcel-parcel and parcel-wall repulsive force 
based on the distinct element method of Cundall and Strack (1979). The parcel-parcel repulsive force $\mathbf{F}_{\mathrm{pj}}^{\mathrm{p}}$ on parcel $\mathrm{p}$ due to collision with parcel $\mathrm{j}$ is given by

$$
\begin{aligned}
& \mathbf{F}_{\mathrm{pj}}^{\mathrm{P}}= \begin{cases}0, & \text { for } \mathrm{d}_{\mathrm{pj}} \geq\left(\mathrm{R}_{\mathrm{ep}}+\mathrm{R}_{\mathrm{ej}}+\rho\right) \\
\left(\mathrm{k}_{\mathrm{c}} \delta_{\mathrm{pj}}^{3 / 2}-\eta_{\mathrm{c}}\left(\mathbf{u}_{\mathrm{p}}-\mathbf{u}_{\mathrm{j}}\right) \cdot \mathbf{n}_{\mathrm{pj}}\right) \mathbf{n}_{\mathrm{pj}} & \text { for } \mathrm{d}_{\mathrm{pj}}<\left(\mathrm{R}_{\mathrm{ep}}+\mathrm{R}_{\mathrm{ej}}+\rho\right)\end{cases} \\
& \delta_{\mathrm{pj}}=\left(\mathrm{R}_{\mathrm{ep}}+\mathrm{R}_{\mathrm{ej}}+\rho\right)-\mathrm{d}_{\mathrm{pj}} \\
& \mathbf{F}_{\mathrm{jp}}^{\mathrm{P}}=-\mathbf{F}_{\mathrm{pj}}^{\mathrm{P}}
\end{aligned}
$$

where $d_{p j}$ is the distance between the centers of the $p^{\text {th }}$ and $j^{\text {th }}$ parcels, $\mathbf{n}_{\mathrm{pj}}$ is the unit vector from the center of parcel $j$ to that of parcel $p, \rho$ is the force range, $k_{c}$ is a stiffness parameter and $\eta_{\mathrm{c}}$ is the damping parameter. Tsuji, Kawaguchi and Tanaka (1993) used the following expression to estimate the damping parameter

$$
\begin{aligned}
& \eta_{\mathrm{c}}=2 \alpha \sqrt{\frac{\mathrm{Mk}_{\mathrm{c}}}{1+\alpha^{2}}} \\
& \alpha=-\ln e / \pi
\end{aligned}
$$

where $\mathrm{M}$ is the mass of the particle (or parcel in our case) and e is the coefficient of restitution. The parcel-wall repulsive force $\mathbf{F}_{\mathrm{pw}}^{\mathrm{W}}$ on parcel $\mathrm{p}$ due to collision with wall $\mathrm{w}$ is given by

$$
\begin{aligned}
& \mathbf{F}_{\mathrm{pw}}^{\mathrm{w}}= \begin{cases}0, & \text { for } \mathrm{d}_{\mathrm{pw}} \geq\left(\mathrm{R}_{\mathrm{ep}}+\rho\right) \\
\left(\mathrm{k}_{\mathrm{c}} \delta_{\mathrm{pw}}^{3 / 2}-\eta_{\mathrm{c}}\left(\mathbf{u}_{\mathrm{p}}\right) \cdot \mathbf{n}_{\mathrm{pw}}\right) \mathbf{n}_{\mathrm{pw}} & \text { for } \mathrm{d}_{\mathrm{pw}}<\left(\mathrm{R}_{\mathrm{ep}}+\rho\right)\end{cases} \\
& \boldsymbol{\delta}_{\mathrm{pw}}=\left(\mathrm{R}_{\mathrm{ep}}+2 \rho\right)-\mathrm{d}_{\mathrm{pw}}
\end{aligned}
$$


where $d_{p w}$ is the distance between the center of parcel $p$ and the wall $w$ and $\mathbf{n}_{p w}$ is the unit vector from the wall $\mathrm{w}$ to center of parcel $\mathrm{p}$. The total collision force $\mathbf{F}_{\mathrm{cp}}$ on parcel $\mathrm{p}$ is then given by

$$
\mathbf{F}_{\mathrm{cp}}=\sum_{\substack{\mathrm{j}=1 \\ \mathrm{j} \neq \mathrm{p}}}^{\mathrm{N}} \mathbf{F}_{\mathrm{pj}}^{\mathrm{p}}+\sum_{\substack{\mathrm{w}=\mathrm{all} \\ \text { walls }}} \mathbf{F}_{\mathrm{pw}}^{\mathrm{W}}
$$

where $\mathrm{N}$ is the total number of parcels. The corresponding acceleration of the parcel (which is the same as the acceleration of the particles belonging to that parcel) is obtained by dividing the collision force by the total mass $\left(\mathrm{N}_{\mathrm{p}} \rho_{\mathrm{p}} \mathrm{V}_{\mathrm{p}}\right)$ of that parcel. Other schemes (e.g. Glowinski et al. 1999, Hu, N. Patankar 2000 and Hu, N. Patankar \& Zhu 2000) that prevent particles (or parcels in our case) from overlapping with each other and the walls may also be used instead of the above.

The collision model is ad hoc and plays a weak role in most regions where interesting flow behavior is observed. The system of equations is strictly valid only in the low Bagnold number regime. Use of such collision schemes are the most practical way of computing dense particulate flows by the direct numerical simulation approaches (e.g. $\mathrm{Hu}$ and N. Patankar 2000, Hu et al. 2000, Johnson and Tezduyar 1997, Glowinski et al.1999, N. Patankar et al. 2000) as well.

Modeling problems, where the particle collisions as well as the fluid flow play an important role in determining the observed behavior, would require a numerical method that combines our current LNS scheme with the Distinct Element Method of Cundall and Strack (1979) for collision dominant flows. Our current method is close to such a computational scheme. Development of a LNS-DEM method is the subject of our future effort. Such an approach will be better for the modeling of dense gas-solid flows.

\subsection{Coupled fluid-particle equations}

We solve the particle momentum equation coupled with the fluid phase equations of motion. The fluid density and viscosity are constant. We use the following first-order temporal discretization of the particle momentum equation 


$$
\mathbf{u}_{\mathrm{p}}^{\mathrm{n}+1}=\frac{1}{\left(1+\mathrm{D}_{\mathrm{p}} \Delta \mathrm{t}\right)}\left[\mathbf{u}_{\mathrm{p}}^{\mathrm{n}}+\mathrm{D}_{\mathrm{p}} \Delta \mathrm{t} \mathbf{u}_{\mathrm{fp}}^{\mathrm{n}+1}-\frac{\Delta \mathrm{t} \nabla \mathrm{p}_{\mathrm{p}}^{\mathrm{n}+1}}{\rho_{\mathrm{p}}}+\Delta \mathrm{t} \mathbf{A}_{\mathrm{bp}}^{\mathrm{n}+1}\right],
$$

where

$$
\mathbf{A}_{\mathrm{bp}}^{\mathrm{n}+1}=\left(1-\frac{\rho_{\mathrm{f}}}{\rho_{\mathrm{p}}}\right) \mathbf{g}+\mathbf{A}_{\mathrm{cp}}^{\mathrm{n}+1} .
$$

Time discretization of (4) and appropriate rearrangement of the terms after using (21) results in the following momentum equation for the fluid phase

$$
\begin{aligned}
& \frac{\rho_{\mathrm{f}} \theta_{\mathrm{f}}^{\mathrm{n}+1}}{\Delta \mathrm{t}} \mathbf{u}_{\mathrm{f}}^{\mathrm{n}+1}+\mathrm{D}_{\mathrm{f}} \mathbf{u}_{\mathrm{f}}^{\mathrm{n}+1}+\mathfrak{I} \mathbf{u}_{\mathrm{f}}^{\mathrm{n}+1}=-\left(1-\mathrm{C}_{\mathrm{pr}}\right) \nabla \mathrm{p}^{\mathrm{n}+1}+\frac{\rho_{\mathrm{f}} \theta_{\mathrm{f}}^{\mathrm{n}}}{\Delta \mathrm{t}} \mathbf{u}_{\mathrm{f}}^{\mathrm{n}} \\
& +\left(\nabla \mathbf{u}_{\mathrm{f}}^{\mathrm{n}}\right) \cdot \nabla\left(\theta_{\mathrm{f}}^{\mathrm{n}+1} \mu^{*}\right)-\left(\nabla \cdot \mathbf{u}_{\mathrm{f}}^{\mathrm{n}}\right) \nabla\left(\theta_{\mathrm{f}}^{\mathrm{n}+1} \mu^{*}\right)+\nabla\left(\theta_{\mathrm{f}}^{\mathrm{n}+1} \mu^{*} \nabla \cdot \mathbf{u}_{\mathrm{f}}^{\mathrm{n}}\right) \\
& +\frac{1}{\mathrm{~V}_{\mathrm{ij} \mathrm{k}}} \sum_{\mathrm{p}}\left[\frac{\left.\rho_{\mathrm{p}} \mathrm{V}_{\mathrm{p}} \mathrm{N}_{\mathrm{p}} \mathrm{S}_{\mathrm{ijk}}\left(\mathbf{x}_{\mathrm{p}}^{\mathrm{n}+1}\right) \mathrm{D}_{\mathrm{p}}\left(\mathbf{u}_{\mathrm{p}}^{\mathrm{n}}+\Delta \mathrm{t} \mathbf{A}_{\mathrm{bp}}^{\mathrm{n}+1}\right)\right], \forall \text { grid node ijk },}{\left(1+\mathrm{D}_{\mathrm{p}} \Delta \mathrm{t}\right)}\right.
\end{aligned}
$$

where

$$
\mathrm{C}_{\mathrm{pr}}=\frac{1}{\mathrm{~V}_{\mathrm{ijk}}} \sum_{\mathrm{p}}\left[\frac{\mathrm{V}_{\mathrm{p}} \mathrm{N}_{\mathrm{p}} \mathrm{S}_{\mathrm{ijk}}\left(\mathbf{x}_{\mathrm{p}}^{\mathrm{n}+1}\right)}{\left(1+\mathrm{D}_{\mathrm{p}} \Delta \mathrm{t}\right)}\right] \text {, for any grid node ijk }
$$

and

$$
\mathrm{D}_{\mathrm{f}}=\frac{1}{\mathrm{~V}_{\mathrm{ijk}}} \sum_{\mathrm{p}}\left[\frac{\rho_{\mathrm{p}} \mathrm{V}_{\mathrm{p}} \mathrm{N}_{\mathrm{p}} \mathrm{S}_{\mathrm{ijk}}\left(\mathbf{x}_{\mathrm{p}}^{\mathrm{n}+1}\right) \mathrm{D}_{\mathrm{p}}}{\left(1+\mathrm{D}_{\mathrm{p}} \Delta \mathrm{t}\right)}\right] \text {, for any grid node ijk. }
$$

$\mathfrak{I}$ is the convection-diffusion operator whose operation on any vector $\mathbf{v}$ is given by

$$
\mathfrak{I} \mathbf{v}=\nabla \cdot\left\{\left(\rho_{\mathrm{f}} \theta_{\mathrm{f}}^{n+1} \mathbf{u}_{\mathrm{f}}^{\mathrm{n}}-\theta_{\mathrm{f}}^{n+1} \mu^{*} \nabla\right) \mathbf{v}\right\}
$$


A "half implicit" expression, $\nabla \cdot\left(\rho_{\mathrm{f}} \theta_{\mathrm{f}}^{\mathrm{n}+1} \mathbf{u}_{\mathrm{f}}^{\mathrm{n}} \mathbf{u}_{\mathrm{f}}^{\mathrm{n}+1}\right)$, is used for the convection term. This expression is first-order accurate. Fluid phase velocity $\mathbf{u}_{\mathrm{f}}^{\mathrm{n}}$ in the convection term can be replaced by a second-order accurate expression given by $2 \mathbf{u}_{\mathrm{f}}^{\mathrm{n}}-\mathbf{u}_{\mathrm{f}}^{\mathrm{n}-1}$ (Turek 1996).

Equation (22) along with the continuity equation of the fluid phase are solved using a Chorin-type fractional-step method (Chorin 1968). We use a non-staggered cartesian grid to solve the fluid phase equations (Rhie and Chow 1982). The cartesian velocity components and pressure are defined at the center of the control volume. Velocities are also stored at the mid-point of their corresponding faces of the control volume. The numerical algorithm will be presented next.

\subsection{Numerical algorithm}

We use explicit update of the particle positions. Motion of each particle type represents the motion of the parcel it belongs to. In our numerical algorithm we first solve for particle positions. This is followed by a solution of the fluid phase equations coupled with the particle momentum equations.

\subsubsection{Explicit update of particle position}

Given the solution at the end of $n$ time-steps i.e. given $\mathbf{u}_{\mathrm{p}}^{\mathrm{n}}, \mathbf{A}_{\mathrm{p}}^{\mathrm{n}}, \mathbf{x}_{\mathrm{p}}^{\mathrm{n}}$ for particles in all the parcels, compute $\mathbf{x}_{\mathrm{p}}^{\mathrm{n}+1}$ by the following procedure:

For particles in all the parcels:

Set $\mathbf{x}_{\mathrm{p}}^{\mathrm{n}+1,0}=\mathbf{x}_{\mathrm{p}}^{\mathrm{n}}$.

do $\mathrm{k}=1, \mathrm{~K}$

$$
\mathbf{x}_{\mathrm{p}}^{*_{\mathrm{n}+1, \mathrm{k}}}=\mathbf{x}_{\mathrm{p}}^{\mathrm{n}+1, \mathrm{k}-1}+\frac{\Delta \mathrm{t}}{\mathrm{K}}\left(\mathbf{u}_{\mathrm{p}}^{\mathrm{n}}+\frac{\Delta \mathrm{t}}{2} \mathbf{A}_{\mathrm{p}}^{\mathrm{n}}\right)
$$




$$
\mathbf{x}_{\mathrm{p}}^{\mathrm{n}+1, \mathrm{k}}=\mathbf{x}_{\mathrm{p}}^{*_{\mathrm{n}+1, \mathrm{k}}}+\left(\frac{\mathbf{A}_{\mathrm{cp}}\left(\mathbf{x}_{\mathrm{p}}^{\mathrm{n}+1, \mathrm{k}-1}\right)+\mathbf{A}_{\mathrm{cp}}\left(\mathbf{x}_{\mathrm{p}}^{*_{\mathrm{n}+1, \mathrm{k}}}\right)}{2}\right) \frac{(\Delta \mathrm{t})^{2}}{2 \mathrm{~K}^{2}}
$$

enddo

Set $\mathbf{x}_{\mathrm{p}}^{\mathrm{n}+1}=\mathbf{x}_{\mathrm{p}}^{\mathrm{n}+1, \mathrm{~K}}$. Calculate $\theta_{\mathrm{s}}^{\mathrm{n}+1}$ by using (11).

Set

$$
\mathbf{A}_{\mathrm{cp}}^{\mathrm{n}+1}=\frac{2}{(\Delta \mathrm{t})^{2}}\left(\mathbf{x}_{\mathrm{p}}^{\mathrm{n}+1}-\mathbf{x}_{\mathrm{p}}^{\mathrm{n}}-\left(\mathbf{u}_{\mathrm{p}}^{\mathrm{n}}+\frac{\Delta \mathrm{t}}{2} \mathbf{A}_{\mathrm{p}}^{\mathrm{n}}\right) \Delta \mathrm{t}\right) .
$$

Here, $\Delta \mathrm{t}$ is the time-step. In this step the effective time-step for particle position update is reduced if the value of $\mathrm{K}$ is greater than one. In our numerical simulations values of $\mathrm{K}$ between 1 and 5 are used. A similar particle position update scheme was used by $\mathrm{N}$. Patankar, Singh, Joseph, Glowinski and Pan (1999) in their direct numerical simulation method for fluid-particle mixtures.

\subsubsection{Numerical scheme for fluid phase and particle momentum equations}

The fluid phase and particle momentum equations are solved simultaneously by the following solution procedure:

(1) Given $\mathbf{u}_{\mathrm{f}}^{\mathrm{n}}, \theta_{\mathrm{s}}^{\mathrm{n}}, \theta_{\mathrm{s}}^{\mathrm{n}+1}, \mathbf{u}_{\mathrm{p}}^{\mathrm{n}}$ and $\mathbf{x}_{\mathrm{p}}^{\mathrm{n}+1}$, compute the intermediate velocity $\mathbf{u}_{\mathrm{f}}^{*}$ at the grid nodes by solving:

$$
\begin{aligned}
& \frac{\rho_{\mathrm{f}} \theta_{\mathrm{f}}^{\mathrm{n}+1}}{\Delta \mathrm{t}} \mathbf{u}_{\mathrm{f}}^{*}+\mathrm{D}_{\mathrm{f}} \mathbf{u}_{\mathrm{f}}^{*}+\mathfrak{I}_{\mathrm{f}}^{*}=\frac{\rho_{\mathrm{f}} \theta_{\mathrm{f}}^{\mathrm{n}}}{\Delta \mathrm{t}} \mathbf{u}_{\mathrm{f}}^{\mathrm{n}} \\
& +\left(\nabla \mathbf{u}_{\mathrm{f}}^{\mathrm{n}}\right) \cdot \nabla\left(\theta_{\mathrm{f}}^{\mathrm{n}+1} \mu^{*}\right)-\left(\nabla \cdot \mathbf{u}_{\mathrm{f}}^{\mathrm{n}}\right) \nabla\left(\theta_{\mathrm{f}}^{\mathrm{n}+1} \mu^{*}\right)+\nabla\left(\theta_{\mathrm{f}}^{\mathrm{n}+1} \mu^{*} \nabla \cdot \mathbf{u}_{\mathrm{f}}^{\mathrm{n}}\right) \\
& +\frac{1}{\mathrm{~V}_{\mathrm{ijk}}} \sum_{\mathrm{p}}\left[\frac{\rho_{\mathrm{p}} \mathrm{V}_{\mathrm{p}} \mathrm{N}_{\mathrm{p}} \mathrm{S}_{\mathrm{ijk}}\left(\mathbf{x}_{\mathrm{p}}^{\mathrm{n}+1}\right) \mathrm{D}_{\mathrm{p}}}{\left(1+\mathrm{D}_{\mathrm{p}} \Delta \mathrm{t}\right)}\left(\mathbf{u}_{\mathrm{p}}^{\mathrm{n}}+\Delta \mathrm{t} \mathbf{A}_{\mathrm{bp}}^{\mathrm{n}+1}\right)\right], \forall \text { grid node ijk },
\end{aligned}
$$


where all the coefficients and source terms in (28) are calculated based on the latest known solution. These values are then considered to be constant during the given time step.

We solve (28) by a block-correction-based multigrid method (Sathyamurthy and S. Patankar 1994). This method employs a multilevel correction strategy and is based on the principle of deriving the coarse grid discretization equations from the fine grid discretization equations. The temporal discretization we have used is first-order accurate. We use the power-law upwinding scheme (S. Patankar 1980) for this convectiondiffusion problem giving a first-order discretization in computational space. Boundary value of the intermediate velocity is the same as the velocity specified there. Outflow boundary condition by S. Patankar (1980) is used wherever there is fluid flowing out of the computational domain. A modified boundary condition for the intermediate velocity that is similar to the one used by Kim and Moin (1985) for single phase flow is recommended for higher-order accuracy. The development of a second-order accurate scheme will be undertaken by us shortly.

(2) Given $\mathbf{u}_{\mathrm{f}}^{*}$ at the grid nodes, compute the intermediate velocity $\left(\mathbf{u}_{\mathrm{f}}^{*}\right)_{\mathrm{cf}}$ on cell faces by linear interpolation (Perić, Kessler and Scheuerer 1988). Value of $\left(\mathbf{u}_{\mathrm{f}}^{*}\right)_{\mathrm{cf}}$ on the boundary cell faces is calculated by linear extrapolation of the values of $\mathbf{u}_{\mathrm{f}}^{*}$ at the interior grid nodes. Other upwind interpolation methods such as the QUICK formulation (Leonard 1979) can be used (Zhang, Street and Koseff 1994). We consider only linear interpolation scheme in the present work for this computational step.

(3) Given $\left(\mathbf{u}_{\mathrm{f}}^{*}\right)_{\mathrm{cf}}$ compute $\phi^{\mathrm{n}+1}$. Correction of the cell face velocity is given by

$$
\frac{\left(\rho_{\mathrm{f}} \theta_{\mathrm{f}}^{n+1} \mathbf{u}_{\mathrm{f}}^{\mathrm{n}+1}\right)_{\mathrm{cf}}-\left(\rho_{\mathrm{f}} \theta_{\mathrm{f}}^{n+1} \mathbf{u}_{\mathrm{f}}^{*}\right)_{\mathrm{cf}}}{\Delta \mathrm{t}}=-\left(1-\mathrm{C}_{\mathrm{pr}}\right)_{\mathrm{cf}} \nabla \phi^{\mathrm{n}+1},
$$


where $\phi^{\mathrm{n}+1}$ is a scalar field to be determined and is defined at the grid nodes. $\mathrm{C}_{\mathrm{pr}}$ at a cell face is calculated by linear interpolation. Note that $\phi$ is different from the real pressure. It is not necessary to solve for real pressure to obtain the fluid velocity field and the particle velocities. The equation for $\phi$ is obtained by using (29) in the continuity equation (1). A discrete form of the equation is then given by

$$
\begin{aligned}
& \frac{\delta}{\delta \mathrm{x}}\left(\left(1-\mathrm{C}_{\mathrm{pr}}\right)_{\mathrm{cf}} \frac{\delta}{\delta \mathrm{x}} \phi^{\mathrm{n}+1}\right)+\frac{\delta}{\delta \mathrm{y}}\left(\left(1-\mathrm{C}_{\mathrm{pr}}\right)_{\mathrm{cf}} \frac{\delta}{\delta \mathrm{y}} \phi^{\mathrm{n}+1}\right)+\frac{\delta}{\delta \mathrm{z}}\left(\left(1-\mathrm{C}_{\mathrm{pr}}\right)_{\mathrm{cf}} \frac{\delta}{\delta \mathrm{z}} \phi^{\mathrm{n}+1}\right)= \\
& \frac{1}{\Delta \mathrm{t}}\left\{\frac{\left(\rho_{\mathrm{f}} \theta_{\mathrm{f}}^{\mathrm{n}+1}\right)-\left(\rho_{\mathrm{f}} \theta_{\mathrm{f}}^{\mathrm{n}}\right)}{\Delta \mathrm{t}}\right. \\
& \left.+\frac{\delta}{\delta \mathrm{x}}\left[\left(\rho_{\mathrm{f}} \theta_{\mathrm{f}}^{\mathrm{n}+1} \mathrm{u}_{\mathrm{fx}}^{*}\right)_{\mathrm{cf}}\right]+\frac{\delta}{\delta \mathrm{y}}\left[\left(\rho_{\mathrm{f}} \theta_{\mathrm{f}}^{\mathrm{n}+1} \mathrm{u}_{\mathrm{fy}}^{*}\right)_{\mathrm{cf}}\right]+\frac{\delta}{\delta \mathrm{z}}\left[\left(\rho_{\mathrm{f}} \theta_{\mathrm{f}}^{\mathrm{n}+1} \mathrm{u}_{\mathrm{fz}}^{*}\right)_{\mathrm{cf}}\right]\right\},
\end{aligned}
$$

where $\delta / \delta x, \delta / \delta y$ and $\delta / \delta z$ represent discrete difference operators in the computational space. We use the velocity specified at the boundary while setting (30). Thus $\phi^{\mathrm{n}+1}$ at the boundary is not required to solve (30). We use the block-correction-based multigrid method (Sathyamurthy and S. Patankar 1994) to solve this equation. Value of $\phi^{\mathrm{n}+1}$ in the domain is calculated with respect to the value at some reference point inside the computational space. To obtain $\phi^{\mathrm{n}+1}$ at the boundary we apply (29) at the boundary cell faces where both $\left(\mathbf{u}_{\mathrm{f}}^{\mathrm{n}+1}\right)_{\mathrm{cf}}$ and $\left(\mathbf{u}_{\mathrm{f}}^{*}\right)_{\mathrm{cf}}$ are known. Velocities at the internal cell faces at the end of the present time-step are computed using (29). These cell face velocities are used to calculate the mass flux in the 'half implicit' convection term in the next time step.

(4) Given $\phi^{\mathrm{n}+1}$, compute $\mathbf{u}_{\mathrm{f}}^{\mathrm{n}+1}$ and $\mathbf{u}_{\mathrm{p}}^{\mathrm{n}+1}$. Particle velocities at the end of the present time step are given by

$$
\mathbf{u}_{\mathrm{p}}^{\mathrm{n}+1}=\frac{1}{\left(1+\mathrm{D}_{\mathrm{p}} \Delta \mathrm{t}\right)}\left[\mathbf{u}_{\mathrm{p}}^{\mathrm{n}}+\mathrm{D}_{\mathrm{p}} \Delta \mathrm{t} \mathbf{u}_{\mathrm{fp}}^{*}-\frac{\Delta \mathrm{t} \nabla \phi_{\mathrm{p}}^{\mathrm{n}+1}}{\rho_{\mathrm{p}}}+\Delta \mathrm{t} \mathbf{A}_{\mathrm{bp}}^{\mathrm{n}+1}\right] .
$$


Fluid velocities at the grid nodes are obtained from the cell face velocities by linear interpolation.

\section{Numerical results}

We solve the problems of bimodal sedimentation, inclined sedimentation, gas-solid fluidization and liquid-solid fluidization to verify the numerical scheme.

\subsection{Bimodal sedimentation}

We validate the numerical scheme by comparing calculated sedimentation rates with the values measured in the experiments of Davis, Herbolzheimer and Acrivos (1982) for a bimodal suspension. The sedimentation column in the experiment was vertical, $100 \mathrm{~cm}$ tall and had a square cross-section with each side $5 \mathrm{~cm}$ wide. The calculation domain in our simulations have $\mathrm{x}, \mathrm{y}$ and $\mathrm{z}$ dimensions equal to $5 \mathrm{~cm}, 125 \mathrm{~cm}$ and $5 \mathrm{~cm}$, respectively. Gravity acts in the negative y-direction. The suspending fluid is Newtonian with the density and viscosity being $992 \mathrm{~kg} / \mathrm{m}^{3}$ and $0.0667 \mathrm{~kg} /(\mathrm{m}-\mathrm{s})$, respectively. Particles of two different densities are used in the calculations. The density of the heavy particles is 2990 $\mathrm{kg} / \mathrm{m}^{3}$; their diameters vary uniformly between $177 \mu \mathrm{m}$ to $219 \mu \mathrm{m}$. The density of lighter particles is $2440 \mathrm{~kg} / \mathrm{m}^{3}$ and their diameters range uniformly between $125 \mu \mathrm{m}$ to $150 \mu \mathrm{m}$. The initial concentration of the heavy particles is 0.01 and that of lighter particles is 0.03 . The particles are initially placed randomly with uniform distribution upto a height of 100 $\mathrm{cm}$ of the sedimentation column. We use Model A for interparticle collision and choose $\mathrm{P}_{\mathrm{s}}=100 \mathrm{~Pa}, \beta=3$ and $\theta_{\mathrm{cs}}=0.6$.

In order to check the convergence of the numerical scheme we perform two simulations with different grid size, number of parcels and time steps. In Case A there are 10 control volumes in the $\mathrm{x}$ and $\mathrm{z}$ directions and 50 control volumes in the $\mathrm{y}$ direction. There are 9000 parcels of each type giving a total of 18000 parcels. The number of particles in each parcel is chosen so that the total particle volume in each parcel is the same for heavy and light parcel types, respectively. The time step is $0.005 \mathrm{~s}$ and $\mathrm{K}=5$. For 
Case B we double the number of parcels in the domain with 12 control volumes in the $\mathrm{x}$ and $\mathrm{z}$ directions and 60 control volumes in the y direction. The time step is reduced to $0.004 \mathrm{~s}$.

Figure 1 shows the transient interface levels of the two types of particles. Here the comparison is made between the LNS calculations from Cases A and B and the experimental data of Davis et al. (1982). We see that they are in good agreement thus validating the calculations by the present numerical procedure. Figure 2 shows the particle positions at $\mathrm{t}=320 \mathrm{~s}$ calculated from Cases A and B. We see that they are in good agreement.

\subsection{Inclined sedimentation}

We apply our numerical scheme to the inclined sedimentation calculations. Acrivos and Herbolzheimer (1979) performed experiments to calculate the sedimentation rates in inclined columns. Experiments were run with the container tilted at different angles. Following Snider et al. (1998) we perform calculations in a two-dimensional domain. Our code for three-dimensional domains is used to perform calculations in twodimensions. The calculation domain in our simulations have $\mathrm{x}$ and $\mathrm{y}$ dimensions equal to $5 \mathrm{~cm}$ and $60 \mathrm{~cm}$, respectively. There are 32 control volumes in the x-direction and 72 control volumes in the y-direction. Gravity acts at an angle of $35^{\circ}$ with the negative $y$ direction. Suspending fluid properties are the same as bimodal sedimentation. The density of the particles is $2420 \mathrm{~kg} / \mathrm{m}^{3}$; their diameters vary uniformly between $130 \mu \mathrm{m}$ to $142 \mu \mathrm{m}$. The initial concentration of the particles is 0.1 . Initially, the particles are randomly placed with uniform distribution upto a height of $52.33 \mathrm{~cm}$ along the y-axis (the mixture-fluid interface is tilted at an angle to vessel walls). We use Model A for interparticle collision with the same parameters as before. There are 18111 parcels in the calculation domain. The number of particles in each parcel is chosen so that the total particle volume in each parcel is the same. The time step is same as before.

Figure 3 shows the transient interface levels of the particles from experiment (Acrivos and Herbolzheimer 1979) and simulation. We see that they are in good agreement. Figure 4 shows the particle positions at different times. 
The mixture-fluid interface can form wave instabilities similar to those of a fluid flowing down an inclined plane. Herbolzheimer (1983) presented photographs of waves at the interface in inclined sedimentation. Snider et al. (1998) simulated wave instability at the interface. We perform the same simulation as Snider et al. (1998) and reproduce the wave observed by them in their simulations. The fluid viscosity is changed to 0.0188 Pa-s, particle diameter is $132 \mu \mathrm{m}$ and particle density is $2440 \mathrm{~kg} / \mathrm{m}^{3}$. The column inclination is $20^{\circ}$. Particles are filled in the column upto a height of $40 \mathrm{~cm}$. All other parameters are the same as in the inclined sedimentation simulations above. Figure 4 shows the formation of wave on the mixture-fluid interface similar to that reported by Snider et al. (1998). Similar waves were also seen during the initial stages of the inclined sedimentation, at $35^{\circ}$, reported above (Fig. 4a). This is in agreement with the simulation results of Snider et al. (1998).

\subsection{Gas-solid fluidization}

Gidaspow (1994) provides a comprehensive summary of the numerical models of gas-solid fluidized bed systems. Most of the effort has primarily focussed on twodimensional simulation using the Eulerian-Eulerian approach. Witt, Perry and Schwarz (1998) reported a numerical model for predicting bubble formation in a threedimensional bed. Tsuji, Kawaguchi and Tanaka (1993) developed an alternate EulerianLagrangian method that used the distinct element method for the solid phase coupled with the Eulerian equations for the fluid phase. They studied two-dimensional fluidization of particles by a jet of gas at the inlet. Ding and Gidaspow (1990) computed two-dimensional fluidization of particles by a uniform inlet flow using the EulerianEulerian approach. They observed two basic flow patterns which were confirmed through experiments. For taller beds a single bubble formed at the center of the fluidization column whereas for shallow bed two bubbles were formed; one each between the walls and the center of the column. In this paper we simulate gas-solid fluidization using our method for incompressible suspending fluids. We ignore the compressibility of gas and intend to verify that our method is able to simulate the above mentioned qualitative features of gas-solid fluidization. 
We first consider the problem of fluidization by a jet of gas at the inlet. The calculation domain have $\mathrm{x}, \mathrm{y}$ and $\mathrm{z}$ dimensions equal to $0.2 \mathrm{~m}, 0.6 \mathrm{~m}$ and $0.0275 \mathrm{~m}$, respectively. Gravity acts in the negative y-direction. The fluid density and viscosity are $1.205 \mathrm{~kg} / \mathrm{m}^{3}$ and $1.8 \times 10^{-5} \mathrm{~Pa}-\mathrm{s}$, respectively. The particle density is $2500 \mathrm{~kg} / \mathrm{m}^{3}$ and the particle diameter are $500 \mu \mathrm{m}$. The initial concentration of the particles is 0.4 . The particles are initially placed in a regular array at the bottom of the column upto a height of about $0.3 \mathrm{~m}$. We perform two simulations with different grid size, number of parcels and time steps. In Case A there are 10 control volumes in the $\mathrm{x}$ direction, 30 in the $\mathrm{y}$ direction and 5 in the $\mathrm{z}$ direction. There are 9720 parcels arranged in a regular array. There are 1000 particles in each parcel. For Case B we there are 16770 parcels (with 580 particles each) in the domain with 15 control volumes in the $\mathrm{x}$ direction, 36 in the $\mathrm{y}$ direction and 6 in the direction. Gas is injected through an opening $0.04 \mathrm{~m}$ wide at the center of column inlet with a superficial velocity of $9 \mathrm{~m} / \mathrm{s}$.

We use Model B for interparticle collision. Our choice of the collision parameters for Model B is entirely empirical when we consider the motion of parcels with large number of particles. We have recently learnt that such grouping of particles is also done when the distinct element method of Cundall and Strack (1979) is applied to the simulation of sand or snow avalanche. To our knowledge there is no theory that estimates or recommends the collision parameters for such group of particles. In applications such as the fluidized beds we expect that the hydrodynamic force on the particles dominate. Hence the choice of collision parameters should not have a major effect on the overall motion of the particles as long as they do not keep the particles too separated. This was confirmed by our own testing as well as the recent results from the direct numerical simulation fluidparticle mixtures within our group where similar collision schemes are implemented to avoid particles from overlapping. For the problem at hand we found that the parameters used by Tsuji, Kawaguchi and Tanaka (1993) did well in keeping the parcels from overlapping. This was not surprising since our parcels are around the same size and mass as the particles in their simulations. We have $\mathrm{k}_{\mathrm{c}}=800$ and $\eta_{\mathrm{c}}=0.018$ in SI units. $\eta_{\mathrm{c}}$ was estimated by (18). $\rho=0.075 \times 2 \mathrm{R}_{\text {ep }}$ was used. Cundall and Strack (1979) proposed that $\Delta \mathrm{t}<2 \sqrt{\mathrm{M} / \mathrm{k}_{\mathrm{c}}}$ be used for stable calculation with a scheme based on explicit update of 
particle positions. We use $\Delta \mathrm{t}=2 \times 10^{-4}$ for Case A and reduce the time step by half for Case B. In both cases we use $\mathrm{K}=1$.

Figure 6 compares the particle configuration from Cases A and B at two times. We see that they are in good agreement. Figure 6a shows a three-dimensional view of the configuration. Figure 7 shows the particle configurations calculated by Case A at later times. It is seen that as the first bubble rises away from the jet inlet there is a tendency to contain solids at their rear center. These solids are further brought into the bubble by the jet that moves faster than the bubble. The jet velocity is not large enough to split the bubble which eventually rises to the top and breaks up. This is in good qualitative agreement with the experiments.

We next simulate a case with uniform inlet velocity. All the parameters are same as Case A of the jet fluidization. Instead of a jet we now have a uniform superficial inlet velocity of $1.8 \mathrm{~m} / \mathrm{s}$. Figure 8 shows the particle configurations at different times. We see that a bubble is formed at the center of the column that rises and breaks at the top. Another simulation was done by increasing the column width to $0.4 \mathrm{~m}$. The number of control volumes in the $\mathrm{x}$ direction are increased to 20 . The number of parcels are also consequently doubled to 19440 . Gas was uniformly injected at the inlet at a velocity of $1.8 \mathrm{~m} / \mathrm{s}$. All the other parameters were kept the same. Figure 9 shows the particle configurations at different times in the wider column. It is seen that there are two bubbles formed in the column as a consequence of increasing its width (or making it shallow). This agrees with what has been observed in experiments.

\subsection{Liquid-solid fluidization}

Liquid-solid fluidized beds in general are known to adopt homogeneous or particulate behavior. In homogeneous fluidization the suspension is mostly uniform and becomes more dilute as the fluidizing velocity is increased. Inhomogeneities in liquid fluidized beds were reported by Cairns and Prausnitz (1960). Hassett (1961a, 1961b) reported three types of flow patterns namely homogeneous fluidization, horizontal striations of low density bands that propagate upwards and lastly breakup of horizontal striations and formation of completely void mushroom shaped bubbles. Further studies in agreement 
with these observations were also reported (see Gibilaro, Hossain and Foscolo 1986 and reference therein). In this paper we compare our numerical results with some of the experimental observations of Gibilaro, Hossain and Foscolo (1986) (henceforth referred to as GHF).

GHF studied the fluidization of zirconia particles in ambient water. They observed that small particle system $(550 \mu \mathrm{m})$ essentially had particulate behavior throughout the expansion range. The $2300 \mu \mathrm{m}$ particles behaved differently; a smooth particulate expansion stage at low fluid volume fractions (voidages) was followed by the development of horizontal upward propagating high voidage bands. At higher velocities (and higher voidages) the voidage bands broke up to form distinct bubbles.

We reproduce these results in our simulation. The fluid density and viscosity are 998 $\mathrm{kg} / \mathrm{m}^{3}$ and $1.009 \times 10^{-3}$ Pa-s, respectively. The particle density is $3810 \mathrm{~kg} / \mathrm{m}^{3}$ (same as zirconia) and their diameter is $2300 \mu \mathrm{m}$. Each parcel has 10 particles. The time step is $0.001 \mathrm{~s}$. The domain size and all the other parameters are the same as that for gas fluidization with uniform inlet velocity. In the present simulation the inlet liquid velocity is varied so that the bed expands to different voidages. Figure 10 shows the particle configuration at different times at an average voidage of 0.53 . We see the formation of horizontal voids that propagate upwards. This is in agreement with the observation of GHF. For voidages less than 0.5 we did not see the formation of such bands. We essentially observed a uniform suspension in fluidized state. Figure 11 shows the particle configuration at different times for an average voidage $>0.6$ (approximately 0.67 ). It is seen that the horizontal voidage bands breakup to form distinct bubbles which is in agreement with the experimental findings of GHF. We also simulate the fluidization of zirconia particles with a diameter of $550 \mu \mathrm{m}$ (751 particles per parcel). We simulated the fluidization of these particles upto a voidage of 0.77 but did not see any prominent aggregate behavior (Figure 12). The instability of a uniform fluidized bed was studied by Anderson, Sundaresan \& Jackson (1995), Glasser, Kevrekidis \& Sundaresan (1996, 1997), Foscolo and Gibilaro (1984), Batchelor (1988) and Joseph (1990). In the future, we intend to investigate the instability of a liquid fluidized bed using our numerical simulations. 


\section{Conclusions}

In this paper we report a new numerical scheme for simulation of particulate flows with the following unique features: (a) The fluid-particle momentum equations are solved in an entirely coupled fashion at a given time-step. (b) This coupled system is solved by a pressure based fractional step scheme for fast computations at each time-step. Traditionally, the pressure based Chorin-type fractional step schemes are devised for constant coefficients in the fluid phase equations. The unique feature of our method is the capability to handle non-constant coefficients in the fluid phase equations and a nonconstant coefficient of the pressure gradient term. (c) The capability to handle dense particulate flows, in challenging simulations such as the fluidization of particulate flows, by the Largangian approach. We show that the parcel approach (instead of an individual particle approach), which reduces the computational cost significantly, captures the basic flow features in fluidization problems.

The numerical scheme is not limited to the particular model used for the effective viscosity of the suspension or the drag and lift forces. It has the flexibility to use different models for these terms which was one of the objectives of this work.

Test cases are simulated to validate the numerical algorithm and its implementation as well as the suitability of the model under various flow conditions. The maximum number of parcels we simulated is 19440. Typically it takes 6-10 s CPU time for the simulation of one time step on a SGI Origin 2000 machine. The maximum memory required for the largest problem solved is around 12-15 MB. Two types of problems are considered for validation: (a) Sedimentation (monomodal, bimodal and inclined) problems where the viscous effects in the fluid phase equations are not important. The same test cases were used by Snider et al. (1998) who neglect the effect of the viscous stress in the fluid phase equations in their numerical approach. The viscous effects are not important in determining the evolution of the height of the sedimentation column in this problem. The numerical scheme was also able to capture the wave instability in inclined sedimentation. (b) Simulations of gas-solid and liquid-solid fluidization are performed. Simulation of a gas-solid bubbling fluidized bed is challenging and it was a breakthrough when the first computation was performed by the System-Science-Software group (Gidaspow 1994, pg. 
152). The earlier computational methods simulated bubbling by a jet of gas and used an inviscid model with an Eulerian-Eulerian approach. Gidaspow (1994, pg. 311) states that inviscid two-phase flow models were able to predict the formation, the growth and the bursting of bubbles in gas fluidized beds with large jets. In such situations the jet establishes the flow pattern. In many industrial applications the gas enters the bed uniformly. In our simulations with uniform gas velocity, we observe that the downward particle motion near the walls sets up the circulation - in agreement with the previous reports (Gidaspow 1994). We were able to establish this flow pattern during the formation of the first bubble with the no-slip condition and the viscous effects near the wall. Simulation results of gas-solid and liquid-solid fluidization are in good agreement with the experimental observations.

Our method is strictly applicable whenever the collision does not play a dominant role in the observed flow behavior. The effect of particle collisions can be important in dense gas-solid flows. The gas-solid flows would therefore be better modeled by a numerical method that combines our current LNS scheme with the Distinct Element Method (DEM) of Cundall and Strack (1979) for collision dominant flows (see e.g. Hoomans, Kuipers, Briels \& Van Swaaij 1996). A fully coupled efficient algorithm based on the numerical technique presented here is the subject of our future investigation.

The way models are tested is through comparisons with experiments. Every model must pass this test. If the prediction of a model disagrees with experiments then it is not valid. If the predictions agree with few experiments it doesn't mean that it is valid either. Our model agrees qualitatively with some experiments; so we are encouraged to look further.

\section{Acknowledgments}

We acknowledge the support from NSF under KDI/NCC grant NSF/CTS-9873236 and STIMLAB.

\section{References}


Acrivos, A. \& Herbolzheimer, E. 1979 Enhanced sedimentation in settling tanks with inclined walls. J. Fluid Mech. 92, 435-457.

Anderson, K., Sundaresan, S. \& Jackson, R. 1995 Instabilities and the formation of bubbles in fluidized beds. J. Fluid Mech. 303, 327-366.

Andrews, M. J. \& O'Rourke, P. J. 1996 The multiphase particle-in-cell (MP-PIC) method for dense particulate flows. Int. J. Multiphase Flow 22, 379-402.

Batchelor, G. K. 1967 An Introduction to Fluid Dynamics. Cambridge University Press, Cambridge.

Batchelor, G. K. 1988 A new theory of the instability of a uniform fluidized bed. J. Fluid Mech. 193, 75-110.

Cairns, E. J. \& Prausnitz, J. M. 1960 Macroscopic mixing in fluidization. AIChE J. 6, 554-560.

Chorin, A. J. 1968 Numerical solution of the Navier-Stokes equations. Math. Comput. 22, 745-762.

Crowe, C. T., Sharma, M. P. \& Stock, D. E. 1977 The particle-source-in cell (PSI-CELL) model for gas-droplet flows. Trans. ASME J. Fluids Engrg. 99, 325-332.

Cundall, P. A. \& Strack, O. D. L. 1979 A discrete numerical model for granular assemblies. Geotechnique 29, 47-65.

Davis, R. H., Herbolzheimer, E. \& Acrivos, A. 1982 The sedimentation of polydisperse suspensions in vessels having inclined walls. Int. J. Multiphase Flow 8, 571-585.

Ding, J. \& Gidaspow, D. 1990 A bubbling fluidization model using kinetic theory of granular flow. AIChE J. 36, 523-537.

Dukowicz, J. K. 1980 A particle-fluid numerical model for liquid sprays. J. Comput. Phys. 35, 229-253.

Foscolo, P. V. \& Gibilaro, L. G. 1984 A fully predictive criterion for transition between particulate and aggregate fluidization. Chem. Engng Sci.39, 1667-1675.

Gauvin, W. H., Katta, S. \& Knelman, F. H. 1975 Drop trajectory predictions and their importance in the design of spray dryers. Int. J. Multiphase Flow 1, 793-816.

Gibilaro, L. G., Di Felice, R., Waldram, S. P. \& Foscolo, P. U. 1985 Generalized friction factor and drag coefficient correlations for fluid-particle interactions. Chem. Engng. Sci. 40, 1817-1823. 
Gibilaro, L. G., Hossain, I. \& Foscolo, P. U. 1986 Aggregate behavior of liquid fluidized beds. Can. J. Chem. Engng. 64, 931-938.

Gidaspow, D. 1994 Multiphase Flow and Fluidization Continuum and Kinetic Theory Descriptions. Academic Press, Boston, MA.

Glasser, B. J., Kevrekidis, F. G. \& Sundaresan, S. 1996 One and two-dimensional traveling wave solutions in gas-fluidized beds. J. Fluid Mech. 306, 183-221.

Glasser, B. J., Kevrekidis, F. G. \& Sundaresan, S. 1997 Fully developed traveling wave solutions and bubble formation in fluidized beds. J. Fluid Mech. 334, 157-188.

Glowinski, R., Pan, T.-W., Hesla, T. I. \& Joseph, D. D. 1999 A distributed Lagrange multiplier/fictitious domain method for particulate flows. Int. J.Multiphase Flow 25, 755-794.

Harris, S. E. \& Crighton, D. G. 1994 Solitons, solitary waves and voidage disturbances in gas-fluidized beds. J. Fluid Mech. 266, 243-276.

Herbolzheimer, E. 1983 Stability of the flow during sedimentation in inclined channels. Phys. Fluids 26, 2043-2045.

Hoomans, B. P. B., Kuipers, J. A. M., Briels, W. J. \& Van Swaaij, W. P. M. 1996 Discrete particle simulation of bubble and slug formation in a two-dimensional gasfluidized bed: A hard-sphere approach. Chem. Engng. Sci. 51, 99-118.

Hu, H. H. 1996 Direct simulation of flows of solid-liquid mixtures. Int. J. Multiphase Flow 22, 335-352.

Hu, H. H., Joseph, D. D. \& Crochet, M. J. 1992 Direct numerical simulation of fluid particle motions. Theoret. Comput. Fluid Dynamics. 3, 285-306.

Hu, H. H. \& Patankar, N. A. 2000 Simulation of particulate flows in Newtonian and viscoelastic fluids, to appear in the International Journal of Multiphase Flow.

Hu, H. H., Patankar, N. A. \& Zhu, M.-Y. 2000 Direct numerical simulations of fluidsolid systems using the Arbitrary-Lagrangian-Eulerian technique, submitted to the Journal of Computational Physics.

Johnson, A. \& Tezduyar, T. 1997 Fluid-particle simulations reaching 100 particles. Research report 97-010, Army High Performance Computing Research Center, University of Minnesota. 
Joseph, D. D. 1990 Generalization of the Foscolo-Gibilaro analysis of dynamic waves. Chem. Engng Sci. 45, 411-414.

Joseph, D. D. \& Lundgren, T. S. 1990 Ensemble averaged and mixture theory equations for incompressible fluid-particle suspensions. Int. J. Multiphase Flow 16, 35-42.

Kim, J. \& Moin, P. 1985 Application of a fractional-step method to incompressible Navier-Stokes equations. J. Comput. Phys. 59, 308-323.

Leonard, B. P. 1979 A stable and accurate convective modeling procedure based on quadratic upstream interpolation. Comput. Methods Appl. Mech. Engrg. 19, 59-98.

Nunziato, J., Passman, S., Givler, C., MacTigue, D. \& Brady, J. 1986 Continuum theories for suspensions. In Advancements in Aerodynamics, Fluid Mechanics and Hydraulics (Proc. ASCE Special Conf., Mineapolis, Minnesota.) (Edited by Arndt, R., Stefan, A., Farrell, C. \& Peterson, S. N.), pp. 465-472.

Patankar, N. A. \& Joseph, D. D. 1999 Lagrangian numerical simulation of particulate flows. Int. J. Multiphase Flow, in review.

Patankar, N. A., Singh, P., Joseph, D. D., Glowinski, R. \& Pan, T.-W. 2000 A new formulation of the distributed Lagrange multiplier/fictitious domain method for particulate flows. Int. J. Multiphase Flow 26.

Patankar, S. V. 1980 Numerical Heat Transfer and Fluid Flow. Hemisphere Publishing Corporation, New York, NY.

Perić, M., Kessler, R. \& Scheuerer, G. 1988 Comparison of finite-volume numerical methods with staggered and colocated grids. Comput. Fluids 16, 389-403.

Rhie, C. M. \& Chow, W. L. 1982 A numerical study of the turbulent flow past an isolated airfoil with trailing edge separation. AIAA-82-0998.

Sathyamurthy, P. S. \& Patankar, S. V. 1994 Block-correction-based multigrid method for fluid flow problems. Numer. Heat Transfer, Part B 25, 375-394.

Snider, D. M., O’Rourke, P. J. \& Andrews, M. J. 1998 Sediment flow in inclined vessels calculated using a multiphase particle-in-cell model for dense particle flows. Int. J. Multiphase Flow 24, 1359-1382.

Thomas, D. G. 1965 Transport characteristics if suspension: VIII. A note on the viscosity of Newtonian suspensions of uniform spherical particles. J. Colloid Sci. 20, 267-277. 
Turek, S. 1996 A comparative study of time-stepping techniques for the incompressible Navier-Stokes equations: from fully implicit non-linear schemes to semi-implicit projection methods. Int. J. Numer. Methods Fluids 22, 987-1011.

Witt, P. J., Perry, J. H. \& Schwarz, M. P. 1998 A numerical model for predicting bubble formation in a 3D fluidized bed. Applied Mathematical Modeling 22, 1071-1080.

Zhang, D. Z. \& Prosperetti, A. 1997 Momentum and energy equations for disperse twophase flows and their closure for dilute suspensions. Int. J. Multiphase Flow 23, 425453.

Zhang, Y., Street, R. L. \& Koseff, J. R. 1994 A non-staggered grid, fractional step method for time-dependent incompressible Navier-Stokes equations in curvilinear coordinates. J. Comput. Phys. 114, 18-33. 


\section{Figure Captions}

Figure 1. Transient interface levels of bimodal batch sedimentation of particles.

Figure 2. Comparison of parcel positions during bimodal batch sedimentation for Cases $\mathrm{A}$ and $\mathrm{B}$ at $\mathrm{t}=320 \mathrm{~s}$.

Figure 3. Transient interface level during inclined sedimentation of particles.

Figure 4. Parcel positions at different times during inclined sedimentation.

Figure 5. Formation of wave at the fluid-mixture interface during inclined sedimentation.

Figure 6. (a) A three-dimensional view of parcel positions during bubbling fluidization of the gas-solid mixture (Case A). (b) Comparison of parcel positions, for Cases A and $\mathrm{B}$, during bubbling fluidization of the gas-solid mixture by a jet, $\mathrm{t}=0.2 \mathrm{~s}$. (c) Comparison at $\mathrm{t}=0.32 \mathrm{~s}$. Parcel diameters are drawn to scale.

Figure 7. Parcel positions at different times during bubbling fluidization of the gas-solid mixture by a jet (Case A). Parcel diameters are drawn to scale.

Figure 8. Parcel positions at different times during bubbling fluidization of the gas-solid mixture by uniform inlet velocity (Tall column). Parcel diameters are drawn to scale.

Figure 9. Parcel positions at different times during bubbling fluidization of the gas-solid mixture by uniform inlet velocity (Shallow column). Parcel diameters are drawn to scale.

Figure 10. Particle configuration at different times during fluidization of the liquid-solid mixture (average voidage $=0.53$ ). Parcel diameters are not to scale.

Figure 11. Particle configuration at different times during fluidization of the liquid-solid mixture (average voidage $=0.67)$. Parcel diameters are drawn to scale.

Figure 12. Uniform fluidization of the liquid-solid mixture with small diameter particles (average voidage $=0.77$. Parcel diameters are drawn to scale. 